\title{
High power coupled midinfrared free-electron-laser oscillator scheme as a driver for up-frequency conversion processes in the x-ray region
}

\author{
M. Tecimer \\ THz-FEL Group, University of Hawaii at Manoã, Honolulu, Hawaii 96822, USA
}

(Received 19 June 2011; published 27 February 2012)

\begin{abstract}
In this paper we present a high-gain free-electron-laser (FEL) oscillator scheme composed of two oscillators that are ideally coupled unidirectionally, with the coupled signal power flowing from the master to the amplifier oscillator. Electron bunches driving the oscillators are in perfect synchronization with the optical pulses building up within the respective cavities. The scheme is applied to a $100 \mathrm{MeV}$ range superconducting energy recovery linac FEL. The computed $\mathrm{mJ}$ level, ultrashort pulse $(<10$ cycles $)$ output in the midinfrared region indicates the potential of the proposed FEL oscillator scheme in driving upfrequency conversion processes in the x-ray region, enabling tunable, high average brightness, attosecond scale coherent soft/hard x-ray sources.
\end{abstract}

DOI: 10.1103/PhysRevSTAB.15.020703

PACS numbers: 41.60.Cr, 42.65.Re, 42.60.Fc, 42.65.Sf

\section{INTRODUCTION}

In the quest for producing tunable, coherent x-ray pulses with femtosecond to attosecond scale durations, high-gain free-electron lasers based on self-amplified spontaneous emission (SASE) and harmonic generation schemes emerged in recent years as the prime candidates to realize powerful x-ray sources covering a spectral range from hundreds of $\mathrm{eV}$ to multiple $\mathrm{keV}$ photon energies. The design concepts of these x-ray free-electron-lasers (FELs) benefit significantly from the recent progresses made in the generation and transport of ultrashort, high brightness electron bunches in conjunction with the advances made in the conventional laser technology in the production of intense, a few-cycle long, low jitter (femtosecond scale), carrier-envelope phase stabilized optical pulses [1]. They utilize laser slicing methods in combination with magnetic chicanes to invoke a femtosecond to attosecond scale microbunching at the fundamental, as in enhanced SASE devices or an efficient harmonic bunching that weakly decreases with increasing harmonic number as in the echo enabled harmonic generation schemes [2-5]. In a different SASE scheme, the FEL interaction is driven directly by femtosecond duration, low emittance, low charge beam pulses that comply with the "short bunch limit" condition leading to the single spike operation [6].

A key aspect in all these $\mathrm{x}$-ray sources is that, due to the extremely short radiation wavelengths and pulse durations targeted, the FEL performance crucially relies on the generation and preservation of extremely bright (ultralow angular and energy spread, high peak current) electron bunches with multi-GeV beam energies and high quality,

Published by the American Physical Society under the terms of the Creative Commons Attribution 3.0 License. Further distribution of this work must maintain attribution to the author(s) and the published article's title, journal citation, and DOI. relatively short period undulators featuring high field amplitude homogeneity and minimized phase errors. As single pass amplifiers, these systems require multiple, lengthy undulator sections (in the total accommodating thousands of periods) along with diagnostic, focusing, and steering components placed in between. The anticipated harvest out of this efforts is promising in terms of coherent $\mathrm{x}$-ray photon fluence ranging from $\sim 10^{9}-10^{13} \mathrm{ph} /$ pulse, peak brightness values amounting to $\sim 10^{26}-10^{33} \mathrm{ph} / \mathrm{s} /(\mathrm{mm} \mathrm{mrad})^{2} / 0.1 \% \mathrm{BW}$, and average brightness to $\sim 10^{18}-10^{25} \mathrm{ph} / \mathrm{s} /(\mathrm{mmmrad})^{2} / 0.1 \% \mathrm{BW}$ [7].

At a different front, smaller laboratory, tabletop highorder harmonic generation (HHG) based sources offer a viable alternative to FELs in the soft $x$-ray and in the near future possibly also in the hard $\mathrm{x}$-ray region for low-average power applications demanding coherent $\mathrm{x}$-ray radiation with subfemtosecond durations. Here, shifting the drive laser wavelengths from near-IR $(\sim 0.8-1.0 \mu \mathrm{m})$ to mid-IR is expected to yield attosecond time scale, $\mathrm{keV}$ range $\mathrm{x}$-ray pulses due to a square of wavelength dependence of the cutoff photon energies in the up-frequency conversion process. The lower conversion efficiency is compensated by taking advantage of more favorable phase matching at longer wavelengths [8]. Recently, a multi-mJ, sub-100 fs mid-IR laser operating at 3.6-3.9 $\mu \mathrm{m}$ has been used to validate the phasematched HHG emission with few tens of attosecond long pulses at the predicted cutoff photon energy of $\sim 0.5 \mathrm{keV}$ [9]. An estimated fluence of approximately $10^{6}-10^{7} \mathrm{ph}$ /laser pulse (within a $1.0 \% \mathrm{BW}$ ) is expected to be obtained with photon energies reaching up multiple keVs by driving the phase-matched HHG at mid-IR wavelengths that are longer than $5 \mu \mathrm{m}[8,9]$. This, however, coincides with a spectral region which suffers from the lack of intense, ultrashort pulsed mid-IR conventional laser sources suitable to drive the targeted HHG process. 
Another approach that shares several common features with HHG, but unlike the latter relies on a stimulated emission process, is the recently identified $\mathrm{x}$-ray parametric amplification (XPA) [10]. The process is driven, similar to $\mathrm{HHG}$, by a strong near-infrared (NIR) [midinfrared (MIR)] fs laser field in the presence of a noble gas. Hereby the HHG signal generated in the same medium serves as the seed for parametric stimulated emission. The process manifests itself in an exponential growth of the $\mathrm{x}$-ray yield with the number of atomic emitters in the gas along with an exponential growth of an initially weak signal until saturation sets in. XPA, being rather in the early study stages with regard to HHG, shows promise for delivering a significant enhancement in $\mathrm{x}$-ray yield by employing a series of optimized amplifier stages [11], with the prospect of overcoming the limitations arising from the low conversion efficiencies inherent to HHG in this spectral range.

To date, multistage optical parametric chirped-pulse amplifier (OPCPA) systems are almost exclusive in providing few-cycle pulses in the mid-IR region with $\mathrm{mJ}$ level pulse energies. A state of the art OPCPA system operating at $\sim 3.6-3.9 \mu \mathrm{m}$ generates multi-mJ, sub-100 fs long compressed mid-IR pulses at a repetition rate of $20 \mathrm{~Hz}$ with the prospect of an increase up to $1 \mathrm{kHz}$ [12]. However, currently used OPA crystals set limitations to extend the above-mentioned performance beyond $\sim 4 \mu \mathrm{m}$. Moreover, it will be a paramount challenge for the coming years to scale the available average power up to 2 orders of magnitude higher values (or more) with the aim to generate few-cycle long mid-IR pulses at megahertz (or higher) repetition rates while sustaining multi-mJ pulse energies; a key route in enhancing the average brightness of phasematched HHG emitted (soft/hard) x-ray photons orders of magnitude for a class of applications that require high average flux while high peak power is not desired. As for the XPA scheme, provided that it lives up to the expectations of enabling notable enhancements in the upfrequency conversion efficiency, synchronously pumped stages of an amplifier chain by multi-mJ level ultrashort laser pulses at high repetition rates will improve the brightness of these coherent, attosecond $\mathrm{x}$-ray sources additional orders of magnitude.

To overcome the limitations arising from the absence of powerful conventional laser sources in the mid-IR region, the use of an FEL is suggested as a driver for HHG [13,14]. The superconducting energy recovery linac (ERL) based NIR/MIR FEL at Jefferson Laboratory (JLab), for instance, is capable of generating $\sim 120 \mu \mathrm{J}, 10$ to 15 cycles short NIR/MIR pulses in the spectral region 1 to $10 \mu \mathrm{m}$ at $\sim 75 \mathrm{MHz}$ repetition rate, operating in quasi-cw mode $[15,16]$. Supporting such high repetition rates, it has the ability of incorporating an enhancement cavity (EC) to boost up the outcoupled micropulse energies. In recent years, the concept of power enhancement of ultrashort laser pulse trains inside a passive cavity is brought forward owing to the locking techniques that enable phase stabilized overlap of wide-bandwidth optical frequency combs excited in master oscillators and enhancement cavities [17-20]. The implementation of an EC (featuring moderate $Q$ values ranging between $\sim 40$ and 50) in conjunction with JLab's ERL mid-IR FEL satisfies in principal the requirements set on a drive laser by the phase-matched HHG process in order to produce attosecond range hard $\mathrm{x}$-ray photons. As elaborated in Ref. [21], the combined system could offer relatively short term a feasible route to verify the predicted cutoff photon energies and the x-ray photon yield obtained by the phase-matched HHG as a function of mid-IR drive laser wavelengths. Because of complications arising from placing the HHG setup within the EC, it might become necessary, however, to cavity dump the amplified mid-IR pulses by means of a semiconductor based fast optical switch [20], constraining hereby the repetition rate of the process to several $\mathrm{kHz}$.

In this paper we present a step further towards achieving a significant enhancement [21] [several orders of magnitude compared to the current status (Refs. $[8,9]$ )] in the average brightness of the coherent, attosecond (soft/hard) $\mathrm{x}$-ray pulses generated by the mentioned up-frequency conversion processes. The focus of our study is on elaborating FEL schemes capable of generating $\mathrm{mJ}$ level (outcoupled), ultrashort ( $\leq 10$ cycles) pulses tunable within the entire mid-IR region (and beyond) with many tens of $\mathrm{MHz}$ (ultimately exceeding $100 \mathrm{MHz}$ ) repetition rates without the need to use external enhancement cavities. (Note that, once the former is demonstrated, the latter can be applied to produce even more energetic pulses with moderate enhancement factors of $\sim 50$ to 100 opening up additional range of strong field applications.) The high repetition rate of the intense mid-IR pulses translates through the up-frequency conversion processes (HHG, XPA) into a high average brightness, attosecond soft/hard $\mathrm{X}$-ray source, tunable (including all generated harmonics) within a spectral range that spans over hundreds of $\mathrm{eV}$ to multiple keV photon energies. In the case of the phasematched $\mathrm{HHG}$ in the $\mathrm{keV}$ region, the expected photon flux at cutoff energies scales to $10^{13}-10^{14} \mathrm{ph} / \mathrm{sec}$ (within $1.0 \%$ $\mathrm{BW}$ ) for $10 \mathrm{MHz}$ operational frequency of the driver FEL.

\section{MILLIJOULE MID-IR FEL OSCILLATOR}

The considered FEL scheme in this study takes advantage of the high powers deposited in the beam (average as well as per bunch) by a $100 \mathrm{MeV}$ range superconducting ERL system operating at high repetition rates. It is capable of generating and transporting a relatively low charge ( $\sim 80 \mathrm{pC}$ ) beam with $\sim 5 \mathrm{~mm}$ mrad normalized emittance, $0.5 \%$ energy spread $\left(\sigma_{E}\right)$, and $100 \mathrm{fs}\left(\sigma_{z}\right)$ long bunches. Several superconducting ERL systems (or their compact test stages) planned to operate at a repetition rate of $1.3 \mathrm{GHz}$ are fulfilling (or close to fulfill) the requested 
beam characteristics. JLab's ERL has also the ability to operate with nearly the same beam parameters at a lower $\sim 75 \mathrm{MHz}$ repetition rate [22].

The proposed FEL oscillator scheme, shown in Fig. 1, relies on the highly advanced, mature technology of (cryocooled) laser optics developed for high power applications in the spectral region of interest. Along with the optical components, it utilizes two relatively short planar undulator sections, each containing 25 periods in length with a period of $6 \mathrm{~cm}$. The device encompasses two coupled oscillator cavities, the first cavity being the master oscillator, the second one acting as an amplifier (slave) oscillator. Mirror A is the high-reflectivity mirror $(R \sim 0.999)$, mirrors $\mathrm{B}$ and $\mathrm{C}$ are partial reflectors with minimized absorption losses. Mirror B, which couples both cavities, is coated with a high transmission antireflection layer on the side facing the amplifier cavity. Mirror $\mathrm{C}$ is the output coupler. Cavity lengths $L_{r}$ of about 20 to 25 meters are considered in the study. The optical delay line incorporated into the amplifier cavity ensures the proper synchronization between the optical pulse, coupled in and building up within the cavity and the injected electron bunch. It is further used for mode matching at the plane of the undulator entrance. The chicane placed between the cavities transfers the electron beam out of the master oscillator into the amplifier cavity, featuring a very low $R_{56}$. The beam transfer between the cavities could be ideally accomplished by employing achromatic bends.

The proposed system has some resemblance with a previously studied scheme, the injection-locked regenerative klystron amplifier presented in Ref. [23]; however, unlike that scheme where the first oscillator acts as a beam energy modulator and the oscillators are solely locked by the electron beam, in the proposed scheme the locking occurs by both, the electron beam as well as the radiation beam. Here, the electron beam has a larger energy spread resulting from the FEL interaction that took place inside the master oscillator. It has to generate a strong seed signal that is transmitted through the coupler mirror into the amplifier cavity. The coupler mirror, on the other hand, has to feature a high enough reflectivity since it acts at the same time as the high reflector for the amplifier cavity which imposes an additional constraint on the coupling strength. Note that neither an optical isolator is placed between the cavities nor a ring amplifier cavity is considered that would impede the feedback, as they are being used in the injection-locked conventional laser configurations [24].

In order to provide further understanding, key concepts underlying the physics of the proposed scheme are briefly discussed below.

\section{A. Weakly coupled lasers}

In general, coupled laser resonator schemes involve arranging multiple lasers sharing partially transmitting mirrors where the output of each laser (or part of it) is fed into the resonator of the others. The behavior of coupled lasers is strongly determined by the optical path length difference of the resonators involved and the amount of coupling between them. A general method of analyzing the interaction between lasers operating with coupled resonators, that is valid for all values of coupling and for any number of coupled lasers, is based on the passive (cold) composite-resonator eigenmode decomposition of the laser fields [25]. The composite-cavity mode approach allows one to derive the eigenmodes and the resonant frequencies of the entire (combined) system of the coupled cold cavities.

The composite resonator of interest in the presented study is a special case of two coupled lasers in the weak coupling limit (transmission of mirror $B \ll 1.0$ ). In this limit the longitudinal distribution of the intracavity field in each laser is given by the eigenmodes of a single, isolated resonator [25,26]. As shown by Spencer and Lamb in [26], for two weakly coupled atomic lasers a stable frequency (or phase) locking occurs when the cavities are closely tuned to equal lengths; the composite laser cavity lases then at a common frequency range and the lasers involved are mutually injection locked. For the given conditions of coupling, the frequency locking remains stable against perturbations arising from cavity length detuning whose

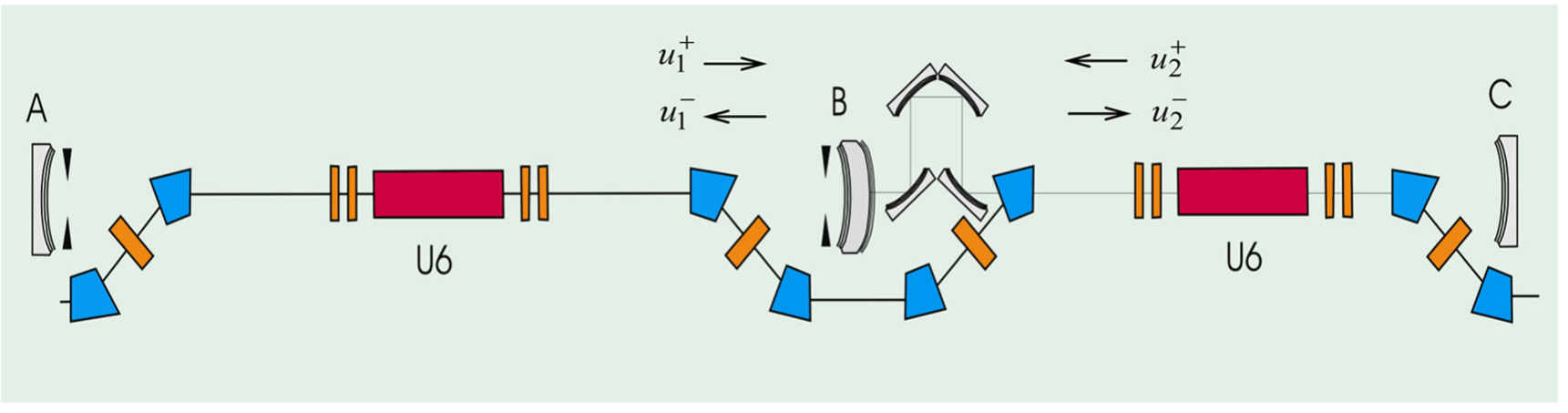

FIG. 1. Schematic layout of the composite high-gain FEL oscillator scheme. $u_{j}^{+}$and $u_{j}^{-}(j=1,2)$ refer to the complex field amplitudes of the incident and reflected radiation fields in the respective cavities with regard to mirror B. This mirror is coated with a high transmission antireflection layer on the side facing the amplifier cavity. 
amplitude is determined by the limits of the locking range [24]. In the studied case, the condition of two coupled lasers tuned to (nearly) equal lengths complies well with the "zero cavity detuning" requirement imposed by the perfectly synchronized FEL oscillator operation where the cavity lengths in Fig. 1 need to be stabilized within a small fraction of the mid-IR wavelengths generated.

The intracavity field analysis of the composite resonator in terms of incident $u_{j}^{+}$and reflected $u_{j}^{-}$complex field amplitudes at the coupling surface can be based on the relations:

$$
\begin{aligned}
& u_{1}^{-}=u_{1}^{+} \sqrt{R_{B}}+u_{2}^{+} \sqrt{1-R_{B}} \\
& u_{2}^{-}=u_{1}^{+} \sqrt{1-R_{B}}-u_{2}^{+} \sqrt{R_{B}},
\end{aligned}
$$

where the indices $j=1,2$ refer to the master and slave oscillators, respectively. $R_{B}$ is the power reflectivity of the coupler mirror. In Eqs. (1) and (2) the choice for determining in which cavity the transmitted and reflected signals will add constructively is somewhat arbitrary since a phase shift of $\pm \pi$ can be introduced in the complex field amplitude $u^{+}$. In the following treatment this choice is made in favor of the amplifier cavity. The complex field amplitude $u_{j}^{+}$is further expressed by the superposition of the (cold) cavities' axial and transverse eigenmodes. Setting $L_{r}=$ $L_{1} \approx L_{2}$, the resonant frequencies of the axial modes in each cavity is given by $\omega_{q}=q c \pi / L_{r}$, where $q$ and $c$ are the axial mode index and speed of light, respectively.

\section{B. FEL oscillator with perfectly synchronized cavity}

An important aspect in our approach is that both oscillators are driven in a single spike modus realized in a short pulse high-gain FEL oscillator with perfectly synchronized optical cavity length ( $\sim$ zero cavity detuning) where the cavity round-trip time of the optical pulse equals the electron bunch interval [27-30]. In this regime that is dominated by high FEL gain, low cavity loss and optical pulse-electron beam slippage effects, the radiation stored in the cavity evolves into an ultrashort, intense spike while keeping a self-similar shape following the saturation. As demonstrated experimentally [27,28], this mode of operation enables also a high FEL extraction efficiency reaching up to many percentages which, along with a highly energetic electron beam, builds the first step towards achieving $\mathrm{mJ}$ level FEL pulses. The dynamics of an FEL operating in this regime is characterized by the so-called short bunch limit [31] stating that the electron bunch length $l_{b}$ scales to the slippage length $l_{s}$ and the cooperation length $l_{c}$ as $l_{b}<l_{s}$ and $l_{b}<2 \pi l_{c}$. Here $l_{s}=N_{u} \lambda_{r}$ and $l_{c}=\lambda_{r} / 4 \pi \rho$; $N_{u}, \lambda_{r}$, and $\rho$ denote the number of the undulator periods, the radiation wavelength, and the FEL parameter [31], respectively. The first scaling indicates that the FEL interaction is slippage dominated. According to the system parameters given in Table I, $\rho$ is $\sim 0.02$; thus, we are
TABLE I. System parameters.

\begin{tabular}{lc}
\hline \hline Electron beam & \\
Energy & $\sim 100 \mathrm{MeV}$ \\
Bunch charge & $80 \mathrm{pC}$ \\
Bunch length $\left(\sigma_{z} \mathrm{rms}\right)$ & $100 \mathrm{fs}$ \\
Normalized emittance & $5 \mathrm{~mm} \mathrm{mrad}$ \\
Energy spread $\left(\sigma_{E} \mathrm{rms}\right)$ & $\sim 0.5 \%$ \\
Repetition rate & $>75 \mathrm{MHz}$ \\
Wiggler & \\
Type & Planar \\
Period & $6 \mathrm{~cm}$ \\
Wiggler parameter $\left(K_{\mathrm{rms}}\right)$ & $\sim 2.6$ \\
Number of periods & 25 \\
Resonators & \\
Wavelength & $\sim 6 \mu \mathrm{m}$ \\
Cavity length & $\sim 20-25 \mathrm{~m}$ \\
Rayleigh range \#1 & $0.7 \mathrm{~m}$ \\
Rayleigh range \#2 & $0.7 \mathrm{~m}$ \\
Cavity $Q$ \#1 & $\sim 35-40$ \\
Cavity $Q \# 2$ & $<10$ \\
\hline \hline
\end{tabular}

dealing with a relatively short cooperation length for the given radiation wavelength. As a result of the high-gain FEL interaction, in the short bunch limit the spike width can become even shorter than the cooperation length. Furthermore, as was demonstrated in Ref. [29], the FEL interaction induces a frequency chirp in the generated spike, making further compression of the pulse duration possible while bringing the pulse width ultimately down to a few cycles. Along with the mentioned high extraction efficiency, the generation of ultrashort ( $<10$ cycles) spikes is an additional factor provided by the presented high-gain FEL oscillator operated at perfect synchronism towards achieving high peak intensities $\left(\sim 10^{14}\right.$ Watts $/ \mathrm{cm}^{2}$ focused to a spot size of $\sim 100 \mu \mathrm{m}$ ) that are sufficient to drive the envisaged up-frequency conversion processes at long mid-IR wavelengths.

For the given system parameters and without additional measures, our estimates presented in the next section indicate, however, that the maximum pulse energies achievable by a single FEL oscillator cavity (even with an optimized extraction efficiency) comes short of meeting the above-mentioned intensity requirements. In the presented study we investigate a composite-resonator structure by incorporating a coupled amplifier cavity that would tolerate relatively large outcoupling losses (despite operating the high-gain FEL at perfect synchronism), be capable of extracting energy out of the "spent" beam to support mJ level outcoupled optical pulses, and maintain largely the spectral/temporal characteristics of the spikes coupled in from the master oscillator. The (power) coupling strength between the cavities is given by $\alpha \approx 1-R_{B}$. The total loss of the respective cavities $\delta_{j}(j=1,2)$ includes $\alpha$ and other losses due to absorption as well as outcoupling losses at mirrors $\mathrm{A}$ and $\mathrm{C}$. 


\section{Injection locking of mode-locked frequency combs}

The fact that the high-gain FEL operation at perfect synchronism enables the generation of ultrashort optical pulses that are propagating with a self-similar shape from one round-trip to the next indicates that the interaction can sustain and amplify a vast number of longitudinal cavity modes excited within an ultrabroad bandwidth (the socalled frequency comb) while a fixed phase relationship (phase locking) between all of them is established. In such a "steady-state mode-locked" operation it is expected that the net dispersion inside the cavity is as close to zero as possible over the broad optical bandwidth. A stable train of mode-locked pulses requires an actively stabilized pulse repetition rate (in our case optical as well as electron pulses) which controls the separation between adjacent comb lines (free spectral range: $\Delta \nu=c / 2 L_{r}$ ) in order to maintain an optimal coupling of the resonant cavity modes to the gain medium, the electron bunches.

Basically a frequency comb is characterized (irrespective of the number of excited cavity modes it encompasses) by two parameters: the repetition frequency of the pulses or the associated free spectral range, as mentioned above, and the carrier-envelope-offset frequency $f_{\mathrm{CEO}}$, which introduces a shift of the entire comb position [32]. The optical frequency of the $q$ th mode in the comb is then given by $\nu_{q}=q \Delta \nu+f_{\mathrm{CEO}}$. The offset $f_{\mathrm{CEO}}$ is the frequency domain manifestation of the phase slip per round-trip of the carrier wave relative to the pulse envelope in the time domain. The difference between the phase velocity of the carrier wave and the group velocity of the envelope gives rise to the carrier-envelope phase (CEP) shift that is susceptible to cavity dispersion. In general, it is possible to control and stabilize the free spectral range (mode spacing) and the offset frequency $f_{\text {CEO }}$ (absolute position of an axial mode in the comb) independently from each other [33]. In fact, mode-locked lasers can operate without active CEP stabilization where fluctuations of $f_{\text {CEO }}$ take place usually on a (much) longer time scale than the round-trip time [33]. However, there is also a growing number of strong field applications in conjunction with the use of enhancement cavities (such as the up-frequency conversion processes mentioned in the Introduction) in which an active control of both degrees of freedom is needed in generating fewcycle near- and mid-IR pulses at high repetition rates by mode-locked lasers.

To successfully injection lock the amplifier cavity with the ultrashort pulse train generated by the master oscillator, the coupled cavities have to be dispersion compensated and their lengths stabilized, conditions that need to be satisfied for the zero cavity detuning FEL operation as well. Here, it is worth noting that the use of a set of matched doubledchirped mirrors not only provides the proper dispersion control but it also offers the needed broad high-reflectivity bandwidth [34]. With these measures, individual modes of the phase-locked frequency comb transmitted from the master oscillator are expected to simultaneously injection lock, as described for the single frequency case in [24,26], the corresponding modes of the frequency comb resonant with the amplifier resonator. It is further expected, as indicated in [19], that the conditions for locking range are valid individually for each cavity mode in the comb of the master oscillator and the closest resonant mode in the amplifier cavity. Here the locking range $\Delta \omega_{\text {lock }}$ between these mode frequencies is given by [24]

$$
\Delta \omega_{\mathrm{lock}} \approx \frac{2 \omega_{r}}{Q_{a}} \sqrt{I_{m} / I_{a}}
$$

where $\omega_{r}$ denotes a resonant mode frequency in the amplifier cavity and $Q_{a}$ is its quality factor (Table I). $I_{m}$ and $I_{a}$ denote the injected input intensity and the outcoupled radiation intensity from the amplifier cavity, respectively (averaged over the number of excited modes). Note that $I_{a}$ is determined for the "free running" laser operation where the slave laser is assumed to operate uncoupled from the master oscillator. In this stand alone operation, having $\sim$ four times lower cavity $Q$ (Table I) the output performance of the second FEL oscillator turns out to be lower than the one provided by the first FEL cavity, despite the larger outcoupling ratio. It is therefore well justified to take $\left(I_{m} / I_{a}\right)^{1 / 2} \geq 1$. Given the low finesse amplifier cavity, one can then expect a fairly stable injection locking of the coupled FEL cavities that is capable of tolerating fairly large excursions in controlling the above mentioned comb parameters. This approximate view does not account for a specific property of the FEL interaction, that namely the gain medium, being modulated in the preceding interaction section can support the injection locking at the initial stages of the radiation buildup in both cavities.

In the following section we seek to find out whether, in view of the nonlinear character of the FEL interaction involving a complex mode competition process and a dynamic gain medium, the phase relations established between the modes along with the spectral bandwidth of the frequency comb generated in the master oscillator will be maintained in the course of the amplification process without noticeable distortion in the amplifier cavity (and in the presence of a mutual coupling, vice versa). In the current treatment of the problem, the emphasis is set on examining the idealized case of decoupling the amplifier cavity's feedback in the reverse direction from the gain medium. In this unidirectional coupling, the radiation power flows from the master into the slave oscillator. In the next step, the latter condition is relaxed allowing a partial bidirectional coupling where a relatively small amount of radiation that is transmitted from the amplifier cavity couples to the gain medium in the master oscillator.

At this point, it is worthwhile to point at a major performance limitation of the presented FEL oscillator scheme. In fact, it concerns also the single stage FEL oscillator operation and arises from the implementation 
of a perfectly synchronized cavity at the wavelength region of interest, ranging from 3 to $8 \mu \mathrm{m}$. Meeting the requirements for the latter will significantly facilitate the injection-locked operation of the coupled laser system as well. The experimental results reported in Refs. [27-29] have been realized by employing a superconducting rf-linac driven short pulse high-gain FEL oscillator in a "perfectly" synchronized cavity to generate few-cycle ultrashort, high peak power radiation at $\sim 20 \mu \mathrm{m}$. Involving significantly shorter electron bunches $\left(\sigma_{z} \sim 100 \mathrm{fs}\right)$ and shorter radiation wavelengths in the mid-IR, a more challenging synchronization of a few femtoseconds is required in order to ensure a stable operation in the presented FEL modus. The latter is feasible with the advent of new synchronization techniques elaborated in x-ray FEL facilities for time resolved experiments that utilize femtosecond to attosecond pulses [7,35], with the tremendous development that took place in recent years in monitoring and stabilizing the synchronization between the subsystems involved in an FEL device to reduce time arrival jitter budget down to a femtosecond time scale [36,37]. On the other hand, although best suited, the operation at a perfectly synchronized cavity is not a prerequisite to the suggested FEL oscillator scheme when performance degradation in terms of peak intensity (smaller pulse energy, higher power fluctuations, longer optical pulse duration) is acceptable for other types of applications.

In the following, the outlined FEL oscillator concept is applied to a compact $100 \mathrm{MeV}, 80 \mathrm{pC}$ low charge ERL driven mid-IR FEL as a potential tool for generating $\mathrm{mJ}$ level, ultrashort ( $\leq 10$ cycles) micropulses with many tens of $\mathrm{MHz}$ to $100 \mathrm{MHz}$ repetition rates, when necessary within lengthy macropulses at low duty cycle. Because of its significance to the phase-matched HHG in the keV region, as pointed out in Ref. [9], the FEL operation at $\sim 6 \mu \mathrm{m}$ will be considered further for the simulations.

\section{SIMULATIONS}

The time dependent, short pulse FEL oscillator code used here is a modified/extended version of the simulation model described in detail in Refs. [38,39]. With the introduced additions it can treat the proposed coupled oscillator structure and an FEL interaction based on injection locking of radiation and electron beams.

In the following we give a brief overview of the theoretical basis underlying the used one-dimensional (1D) and a computation intensive three-dimensional (3D) formulation. The formalism is based on expanding the radiation vector potential $A_{r}$ in terms of transverse eigenmodes of the oscillator cavity:

$$
\begin{aligned}
\vec{A}_{r}\left(\vec{x}_{\perp}, z, t\right)= & \frac{e}{m_{e} c} \sum_{m, n} u_{m n}(z, t) \psi_{m n}\left(\vec{x}_{\perp}, z\right) \\
& \times \exp \left[i\left(k_{o} z-\omega_{o} t\right)\right] \hat{x},
\end{aligned}
$$

where $u_{m n}(z, t)$ is the transverse mode's complex amplitude slowly varying in time and space, $\Psi_{m n}$ refers to the $m n$th transverse eigenmode of the cavity (here we use Hermite-Gaussian modes), $\omega, k_{o}$ are the angular frequency of the resonant carrier wave, its wave number, respectively. The electric and magnetic fields can be derived from the vector potential by $\vec{E}_{r}=-\partial_{t} \vec{A}_{r}$ and $\vec{B}_{r}=\nabla \times \vec{A}_{r}$. With the adopted slowly varying envelope approximation, the FEL radiation field dynamics are described in time and space by the wave equation:

$$
\begin{aligned}
\left(\partial_{z}+\frac{1}{c} \partial_{t}\right) u_{m n}(z, t)= & -\frac{e \mu_{0}}{m_{e} c} \frac{e^{-i\left(k_{0} z-\omega_{0} t\right)}}{2 i k_{z} \Sigma} \\
& \times \int J_{\perp}\left(\vec{x}_{\perp}, z, t\right) \psi_{m n}^{*}(\vec{x}, z) d^{2} \vec{x},
\end{aligned}
$$

where $\Sigma$ denotes the mode area. The transverse current density $J_{\perp}$ in the source term is determined by the sum over single (macro)particle contributions:

$\vec{J}_{\perp}\left(\vec{x}_{\perp}, z, t\right)=-e c \sum_{j} \vec{\beta}_{\perp j} \delta\left[\vec{x}_{\perp}-\vec{x}_{j}(z)\right] \frac{\delta\left[t-t_{j}(z)\right]}{v_{z j}}$,

where $\vec{\beta}_{\perp_{j}}$ is the normalized transverse velocity of the $j$ th particle in the electron bunch, $v_{z j}$ is its longitudinal velocity, and $t_{j}(z)$ is the particle's arrival time at any position $z$ within the interaction region.

As discussed in Ref. [39], in the computational fast 1D approach Eq. (5) is simplified by averaging the source term over radiation and undulator periods, by replacing the discrete sum over $\delta$ functions with a continuous Gaussian density profile, and by carrying out a coordinate transformation to eliminate the partial time derivative. On the other hand, the simplified equations of motion, underlying the particle mover in the numerical algorithm, follow closely the Kroll-Morton-Rosenbluth (KMR) formalism [40]. The radial inhomogeneity of the radiation field experienced by the electrons due to their transverse excursion is accounted for by averaging the source term in the wave equation over the transverse Gaussian density profile of the electron beam [41]. In view of the current densities and beam energies utilized in our case, space-charge forces between the particles are ignored (Compton regime).

The 3D extended code is more involved first in that a multifrequency approach is implemented in addition to the transverse mode expansion in expressing the fields by a properly chosen number of longitudinal cavity modes centered around the resonant frequency $\omega_{0}$ and solving the wave equation, Eq. (5), for the complex amplitude $u_{m n}^{(q)}$ with the corresponding $\omega^{(q)}$ and $k_{z}^{(q)}(q)$ denoting the axial mode index [39] (see also [42,43]). No averaging has been carried out in the source term [Eqs. (5) and (6)]; the radiation field is obtained by summing up the contributions emitted from each particle in the beam. The approach is more appropriate in dealing with broadband, several cycle 
long optical pulses where the carrier envelope starts varying notably on the scale of the wavelength [44]. Furthermore, it allows a detailed description of the optical field amplitude and phase evolution in the coupled laser resonators at the level of individual axial modes (here "supermodes" representing the behavior of a group of neighboring axial cavity modes). In the presence of the undulator magnetic fields and the radiation fields, the 3D Lorentz force equations along with the energy exchange equation have been solved for each particle in conjunction with the evaluation of the radiation fields utilizing the above-mentioned wave equations. The computation intensive 3D code is used for sample cases to verify the assumptions stated in the previous section regarding the injection locking of frequency combs and to find out whether the phase relations between the modes of the comb, that are established in the single stage FEL at perfect synchronism, can be sustained during the amplification process in a coupled FEL oscillator system. Based on the retrieved evolution of the carrier envelope (amplitude and phase), the fast $1 \mathrm{D}$ code is applied extensively in the simulations to optimize the coupling parameters.

The starting point for the simulations is the system parameters given in Table I. Having the beam parameters fixed, the simulations were focused on optimizing the reflection and transmission characteristics of the cavities. The resulting optimization underlines the importance of a low coupling strength $\alpha$ between the cavities in achieving the envisaged performance output. In the targeted FEL regime and the unilaterally coupled laser operation, for the given system parameters, an optimized intensity output accompanied with ultrashort spike generation is found to occur for $\alpha \sim 0.02$. Because of the increasing growth of instabilities (arising mainly from the mode competition) that are observed at the transition to mutual coupling, $\alpha$ is restricted in this regime to values $\geq 0.01$.

\section{A. Unilaterally coupled laser system}

Here the feedback of the amplifier cavity is assumed to be decoupled from the gain medium in the master oscillator. The radiation contribution due to the second term at the right-hand side (rhs) of Eq. (1) (at the beginning only a small fraction of the first term) is prevented from being coupled to the gain medium. The approach reduces the complexity of the problem compared to the case where coupling occurs in both directions. Setting $\alpha=0.02$, the evolution of the seeding pulse energy transmitted into the amplifier cavity is shown in Fig. 2(a). Considering all cavity losses per round-trip, the cavity $Q$ is set to be $\sim 35$. Figures 2(a)-2(c) display the temporal profile and a broadband spectrum of the generated spikes that are inherent to this low loss, ultrashort pulse, high-gain FEL regime [29,30]. Figures 2(d)-2(f) show, for the same setting, the results obtained by using the $3 \mathrm{D}$ nonaveraged
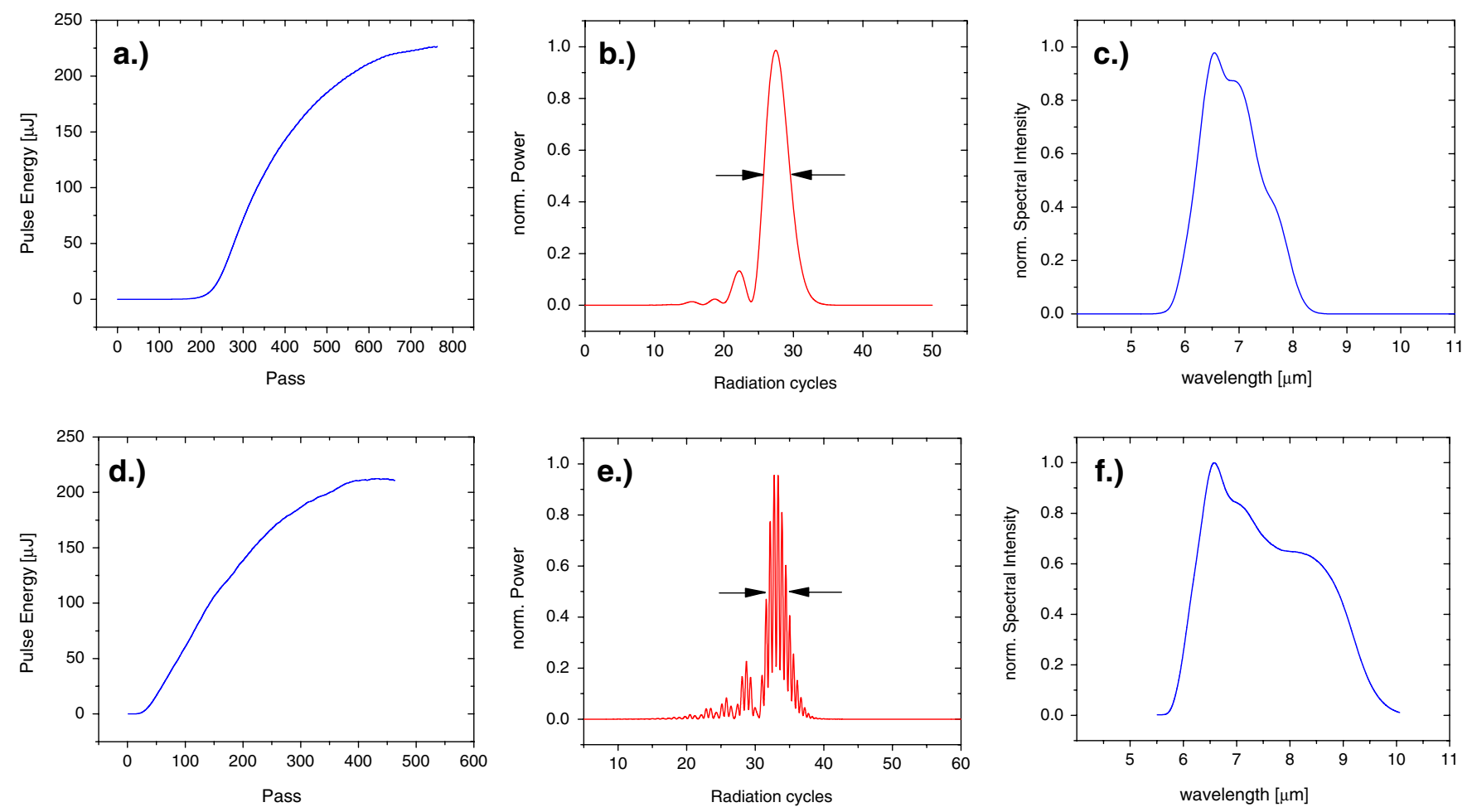

FIG. 2. (a)-(f) Results obtained from 1D code (above row) with $\alpha=0.02$ are contrasted with the ones computed by $3 \mathrm{D}$ modeling for the simulation of seeding pulse energy evolution in (a) and (d), optical pulse temporal profile in (b) and (e), as well as spectrum in (c) and (f), respectively. The computed pulse widths amount to $\sim 4$ cycles (1D) and $\sim 3.5$ cyles (3D nonaveraged, multifrequency code). 
multifrequency modeling where the simulated pulse width is slightly shorter than the one obtained by the 1D code. The spectrum turns out to be significantly broader. The phase of the generated radiation fields exhibits a frequency chirp in the spectrum induced by the FEL interaction (not shown here). The pulses are not Fourier transform limited, the time-bandwidth product deviating notably from $\sim \frac{1}{2}$. A superradiant like start-up and pulse energy growth at the initial stages of the FEL interaction helps to reduce the number of passes until saturation is reached, as shown in Fig. 2(d). Besides 3D related effects, the latter is attributed to the nonaveraged, multifrequency evaluation of the wave equation in conjunction with the start-up noise generated in the initial particle loading [45]. The curves shown in 2(a) and 2(d) reach the steady state with a fairly good agreement in the transmitted pulse energies: $\sim 230 \mu \mathrm{J}$ and $\sim 210 \mu \mathrm{J}$, respectively.

Decoupling the feedback, the FEL dynamics in the master oscillator evolves according to an isolated highgain FEL oscillator operating with perfectly synchronized cavity. The injected optical pulses develop to intense, ultrashort spikes as shown in Figs. 2(a)-2(f). Their fields are additive to the ones circulating and building up in the amplifier cavity. Over the adjustable delay line, a welldefined synchronization with the injected electron beam is established as well. Note that, unlike the newly injected electron bunches into the first undulator on successive round-trips, in the amplifier cavity the electrons enter the interaction section after acquiring a relatively large energy spread due to the past FEL interaction. Here, the beam transport between the oscillators is assumed to be realized by a bypass section consisting of achromatic bends.

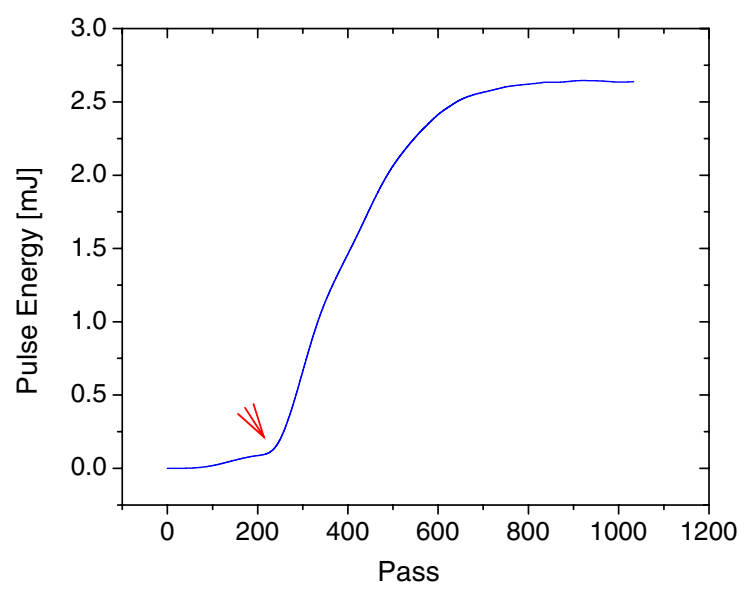

FIG. 3. Pulse energy evolution displaying the output performance of the suggested composite FEL oscillator (unilateral coupling). The arrow refers to the onset of the strong growth accompanied with injection locking of the wideband frequency comb. The coupling strength $\alpha$ is set to 0.02 . The evolution of the seeding input is displayed in Fig. 2(a). Here the (amplifier) cavity $Q \sim 10$ is assumed.

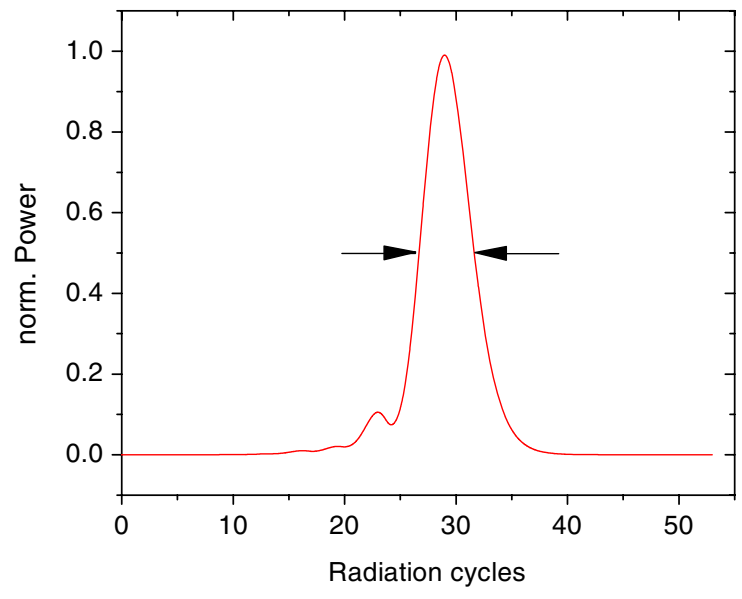

FIG. 4. The temporal profile of the outcoupled ultrashort pulse at FEL saturation.

Figure 3 shows that, once the injection locking of the wideband frequency combs excited in the coupled oscillators sets in, a substantial intracavity power growth starts taking place (indicated with an arrow), despite using a large extraction ratio compared to the master oscillator. At the beginning the transmitted pulse duration is still long and the bandwidth (along with the number of the excited axial cavity modes) is relatively small. Gradually the coupled spike duration shortens, the associated spectrum broadens, and the peak intensity becomes larger as a result of the high-gain FEL interaction at zero cavity detuning. The temporal profile of the pulse extracted from the amplifier oscillator after reaching saturation is displayed in Fig. 4. A comparison with the shape of the injected pulses, shown in Fig. 2(b), reveals that the amplification process discriminates the side peaks and lengthens the pulse duration. The latter occurs moderately, resulting in $\sim$ five cycle long pulses from the initial four cycles. (The simulation results obtained by the nonaveraged, multifrequency code yields a smaller increase in the pulse duration.) The sustained temporal profile of the injected pulses indicates that the established phase locking among the excited comb lines in the master oscillator remains intact without noticeable distortion during the amplification process in the amplifier cavity. The notable result that can be deduced out of this picture is that the injection-locking mechanism described in the previous section remains (to a large extent) valid for the FEL interaction. Note that the described interaction is fundamentally different from other multiple FEL oscillator schemes where the first oscillator stage acts as a modulator of an injection locked (by the electron beam) Klystron amplifier [23]. The dominant locking mechanism in the presented approach is due to the injected radiation fields as test runs have been conducted to evaluate the output performance of the FEL systems by (i) replacing the spent beam (transported into the amplifier cavity) at each round-trip by a fresh electron beam while injecting 
the same radiation fields transmitted from the master oscillator, and (ii) blocking the injection of radiation fields from the master oscillator while only the spent beam was used to drive the FEL interaction in the second cavity at perfect synchronism.

A key factor enabling an efficient radiation power extraction in the injection-locked FEL operation is that a large number of modes excited within a wideband frequency comb can couple phase locked, at the presence of high power levels accumulated inside the cavity, to an ultrashort electron bunch with relatively large energy spread and a broad gain bandwidth. Interacting with the intense, ultrawideband fields, the spent beam's longitudinal phase-space distribution is reorganized, leading effectively to an increased bunching in the amplifier cavity and a high overall FEL extraction efficiency. Hereby, it should be pointed at the involvement of a mechanism that might help to sustain the resonance condition for particles injected into the amplifier cavity, contributing to the favorable bunching process in the amplifier cavity. The effect concerns the synchrotron oscillation behavior exhibited in the presence of the intense optical pulses that leads to radiation pulse shortening (already in the master oscillator), which is indicated previously in high-gain, slippage dominated FEL oscillators operating at perfect synchronism and in the seeded FEL amplifier scheme described in [46], where energy transfer from the intense radiation pulse to the particles at the bottom of the ponderomotive potential well takes place. The longitudinal phase-space evolution of the spent beam at the transport into the amplifier oscillator and at various stages of its interaction with the circulating intracavity fields is subject to further study. This applies also, as a contrast to the latter, for the longitudinal phase-space evolution of the newly injected fresh electron pulses (that are assumed to replace the spent beam) into the amplifier cavity at each round-trip, as in the abovementioned (hypothetical) case. Results of the study will be presented elsewhere.

Further analysis by carrying out curve fittings at various sections of the pulse energy growth versus the respective interaction lengths along the $z$ axis reveals the existence of different scalings with $z$. Starting from the onset of the strong growth region (indicated by the arrow) in the first $\sim 150$ passes the scaling can be approximated by $\sim z^{3 / 2}$ [46-48] rather than by an exponential dependency. At a later stage of the interaction, there is a transition into an exponential dependency which ultimately merges into saturation. Finally, for the given parameters, a gain factor that is larger than ten is achieved with regard to the output of a single oscillator stage, resulting in $\mathrm{mJ}$ level pulse energies at high repetition rates.

\section{B. Partial bidirectional coupling}

The injection-locking mechanism presented in Sec. II C is extended to treat the (partial) bidirectional coupling. The reflected and transmitted field amplitudes (feedback from the amplifier cavity) at mirror B are summed up according to Eq. (1). As the intracavity power buildup in the second cavity exceeds more and more that of the first cavity, the second term at the rhs of Eq. (1) becomes gradually influential for the FEL interaction. The resulting loss (or gain) modulation of the axial modes in conjunction with the mode competition leads to "limit cycle oscillations" in the field intensities that are transmitted through the weak coupling over to the second cavity (and back again and so forth). The studied mutually coupled FEL oscillator system becomes susceptible to instabilities, such as the onset of high intensity fluctuations. The latter turns out to be more pronounced in the preliminary simulations that are carried out by using the nonaveraged, multifrequency modeling of the mutually coupled FEL oscillator system. Thus, an optimized output performance of the proposed scheme entails adopting approaches that would ideally eliminate or at least reduce the coupling of feedback to minimum levels in the reverse direction in conjunction with implementing additional measures such as an active CEP stabilization of the fields evolving in the cavities. On the other hand, another alternative, the injection-locked oscillator configuration that impedes the feedback by employing a ring cavity amplifier (slave laser) [24,49] leads in our case to a considerable performance reduction in terms of extraction efficiency.

The approach suggested here to reduce the feedback in the reverse direction relies on the tight synchronization conditions imposed by the FEL interaction between $100 \mathrm{fs}$ or shorter optical and electron beam pulses in conjunction with effects arising from the applied resonator characteristics. In the following the laser axis (resonatoroptical axis as well as undulator-electron beam axis) of the amplifier cavity (Fig. 1) is assumed to be rotated by a small angle (up to $\sim 50 \mu \mathrm{rad}$ ) at around the center of the coupling mirror B (mirror B remains fixed). The transverse mode center of the injected spike at mirror $\mathrm{B}$ is then located slightly $(\sim 0.5 \mathrm{~mm})$ off axis for the mode excited in the amplifier cavity. However, transported by a set of properly curved (delay line) mirrors it is well matched along the $1.5 \mathrm{~m}$ long interaction section (in terms of transverse mode structure and position) with the transverse mode of the amplifier cavity. Based on the resonator parameters listed in Table I, assuming a transverse misalignment tolerance of $\pm \sim 300 \mu \mathrm{m}$ and an angular misalignment tolerance of $\pm \sim 25 \mu \mathrm{rad}$ between the axis of both transverse modes at waist, that is located at the center of the undulator, the mode coupling efficiency $\mathcal{C}_{00}$ amounts approximately to $90 \%$. Here, the efficiency of coupling the injected pulse into the one circulating in the amplifier cavity is estimated by the projection of the respective fundamental Hermite-Gaussian modes (which does not account for the guiding provided by the highgain FEL medium with a gain length of $\sim 20-25 \mathrm{~cm}$ ): 


$$
\mathcal{C}_{00}\left(z_{w}\right)=\int_{-\infty}^{\infty} \int_{-\infty}^{\infty} \psi_{\text {cir }}\left(\vec{x}_{\perp}, z\right) \psi_{\text {inj }}^{*}\left(\vec{x}_{\perp}, z\right) d x d y
$$

The optical pulse transmitted through the coupler mirror into the master oscillator, on the other hand, arrives at the respective undulator entrance, after being bounced off-axis from the high reflector, mismatched to the established intracavity mode. Having acquired a relatively large transverse displacement and slope (along with a longitudinal delay), its coupling to the gain medium is reduced to a large extent already at the first round-trip. For the given resonator parameters and the assumed $\sim 50 \mu$ rad angle between the resonator axis, the transverse coupling efficiency is reduced down to $\sim 20 \%$. The remaining part experiences additional losses (besides $\delta_{1}$ ) with the help of the apertures placed inside the master oscillator (Fig. 1)

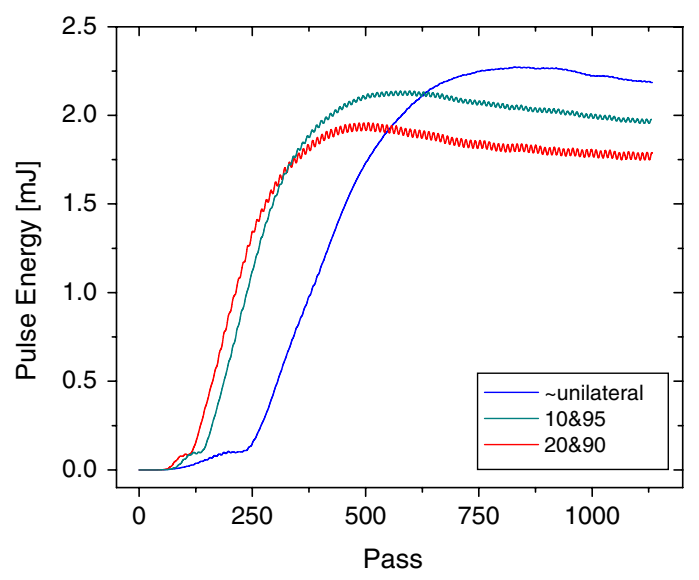

FIG. 5. Outcoupled pulse energy evolution allowing bidirectional coupling to the gain mediums: $\alpha, Q_{1}$, and $Q_{2}$ being set to $0.01, \sim 40$, and $\sim 10$, respectively. The values before and after the ampersand denote the injected seed signal coupling efficiency (in percentage) to the gain medium in the first and the second cavities, respectively. which constitute a loss channel to remove the parasitic radiation from the cavity. Other means can be adopted to cope more efficiently with this feedback radiation which is out of the scope of the current study.

Figure 5 displays the effect of the described partial mutual coupling on the injection-locked amplification process along with the modulation behavior exhibited in the pulse energy evolution according to simulation results obtained by using the $1 \mathrm{D}$ code. The coupling to the gain medium in the reverse direction is restricted to $\sim 2 \times 10^{-3}$ of the radiation power circulating in the amplifier cavity with $\alpha$ being set to 0.01 . The dependency of the outcoupled pulse energy and the amplitude of the limit cycle oscillations on the estimated coupling efficiency of the feedback is illustrated by assuming three different coupling scenarios. The temporal profile shown in Fig. 4, on the other hand, is nearly conserved (along with the phase locking established among the cavity modes in the frequency comb) for the considered cases. An active CEP stabilization is imposed on the radiation pulse evolution in the simulations of the described partial bidirectional coupling. In the absence of the phase stabilization the output performance of the described mutually coupled system deteriorates drastically, exhibiting intensity oscillations accompanied with a strong reduction of the generated pulse energy. In this case, as shown in Fig. 6, a drop in the amount of coupling in the reverse direction leads to an increase in the period and a decrease in the amplitude of the intensity oscillations for the pulses building up in the amplifier cavity. Preliminary simulations (carried out with reduced number of macroparticles representing the electron bunches) that use the nonaveraged, multifrequency modeling indicate that, for the studied parameter setting a drastic improvement in terms of pulse energy increase, damping of instabilities starts occurring only then when a very small fraction of the transmitted feedback is allowed to be superimposed to the fields circulating in the master oscillator [Eq. (1)] and to be coupled to the electron beam.
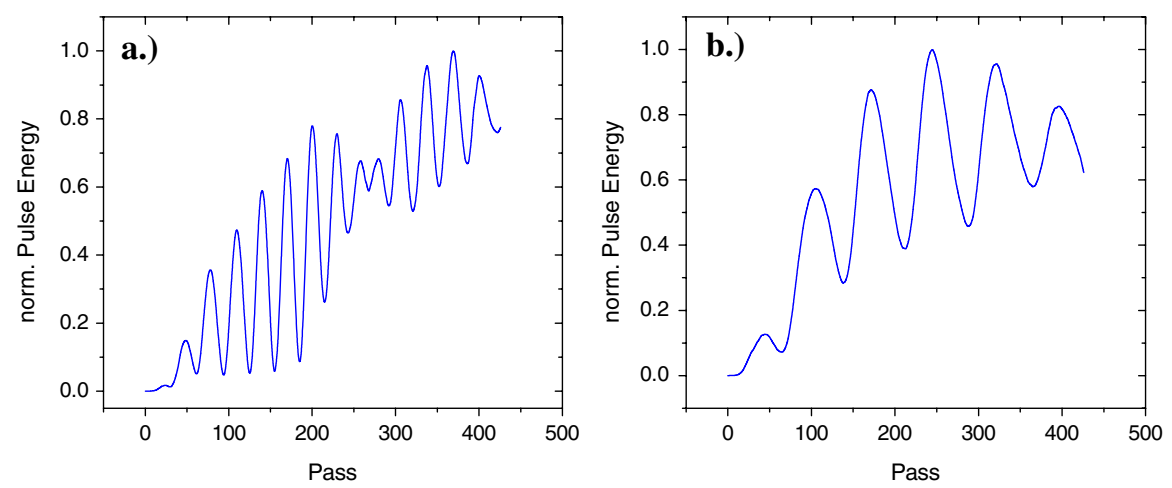

FIG. 6. (a) and (b). Pulse energy evolution (normalized to the maximum) for the asymmetrical bidirectional coupling without carrier envelope phase stabilization: $\alpha, Q_{1}$, and $Q_{2}$ being set to $0.01, \sim 40$, and $\sim 10$, respectively. The coupling ratio of the feedback to the electron beam in the master oscillator amounts to $20 \%$ of $\alpha$ in (a) and only $5 \%$ of $\alpha$ in (b). This ratio is set to be $90 \%$ of $\alpha$ in the amplifier cavity for both cases. 
This implies for the studied parameter range, without introducing additional measures a stable operation of the proposed FEL oscillator scheme seems to be possible only by ensuring a nearly perfect decoupling in the reverse direction ( $\sim$ unilateral operation). It remains therefore interesting to pursue both directions in future work: (i) elaborating further on schemes that enable a nearly $100 \%$ decoupling, including the one suggested in this paper and (ii) exploring further approaches that counteract the coupling induced instabilities in the case of the proposed "asymmetrical" mutual coupling, where the coupling to the gain medium in the reverse direction is restricted to be a small fraction ( $\sim$ some percentages) of $\alpha$.

The nonlinear dynamics of unidirectionally, bidirectionally instantaneous, or delay coupled lasers (mainly semiconductor lasers and their arrays) in the weak and strong coupling regimes has been a subject of intense research previously [50]. A comprehensive model in describing the properties of these systems was developed that is based on the composite-cavity mode approach [25,50], as pointed out in Sec. II. From a universal point of view, the dynamics of coupled nonlinear oscillators (and their networks) have been investigated in very different fields in biology, chemistry, engineering, and physics [51,52]. A more simplified and widely used approach (with regard to the compositecavity mode approach) in describing the behavior of coupled atomic lasers is based on the field equations of a slowly varying complex field amplitude for a single axial mode [53,54]. To the best of the author's knowledge, a coupled laser oscillator system, as described in this paper, has not been reported before. The applied (nonaveraged) multifrequency FEL modeling along with Eqs. (1) and (2) correspond in the weak coupling limit and for nearly identical cavities to the above-mentioned composite-cavity mode approach, apart from the rate equations that describe the dynamics of population inversion in atomic lasers. Note also that, in the implemented modal expansion method the evolution of the coupled mode amplitudes $u_{m n}^{(q)}$ are determined by solving first order (ordinary) differential equations [39].

Finally, it should be also pointed out that a more general multifrequency simulation model for short pulse FELs compared to the adopted Fourier series expansion is the space-frequency representation (Fourier decomposition) of the radiation fields and the current sources in deriving the mode excitation equations in the frequency domain [55] (see also [56]). The three-dimensional numerical implementation of the latter requires even greater computational effort in contrast to the one presented in this paper. On the other hand, dealing with ultrashort, broadband pulses propagating in the presence of dispersive (nonlinear) components/media [57] within the cavity, the space-frequency approach constitutes a straightforward way of tackling the problem without introducing further approximations in the FEL modeling.

\section{Outlook}

In Sec. III A the possibility of injecting fresh electron bunches (by replacing the spent beam transported from the master oscillator) into the amplifier cavity was considered. In simulating the case an additional performance increase in terms of pulse energy was already observed. A different alternative towards achieving higher pulse energies at high repetition rates, however at the cost of the system becoming more complex, is (after compression) the seeding of the intense, ultrashort pulses generated by the proposed coupled oscillator scheme into a single pass high-gain FEL amplifier, similar to the scheme demonstrated in [46].

In the presented study the used simulation model yields, under the described idealized conditions for an injectionlocked FEL amplification of ultrashort, broad bandwidth mid-IR pulses, an exceptionally high extraction efficiency for a rf linac based FEL system reaching up to 25\%-30\% (unilaterally coupled system). A record high conversion efficiency of $\sim 6 \%$ was already demonstrated in a single stage superconducting rf-linac mid-IR FEL oscillator operating at perfectly synchronized cavity as reported in Refs. $[28,30]$. Here, aside from the shortcomings of the employed modeling, it is expected that the computed high FEL efficiency will be reduced under real life conditions, mainly due to imperfections arising from the implementation of a perfectly synchronized cavity in the wavelength region of interest. Nevertheless, the proposed coupled, injection-locked FEL oscillator concept describes a possible route for achieving a remarkable improvement in the output performance of a $100 \mathrm{MeV}$ range ERL mid-IR FEL device, enabling multi-mJ ultrashort pulse energy output. A more comprehensive study is under way that includes the effects of various imperfections (such as jitter in the electron bunch arrival time, uncompensated dispersion of optical components in the cavities) and, as mentioned above, also elaborates on schemes enabling a high efficiency in decoupling the feedback in the reverse direction.

In principal, in order to compensate for a likely reduction in the extraction efficiency, the beam energy can be increased within a range from 100 to $150 \mathrm{MeV}$. In addition, with the available technology there is a realistic margin for an increase in the bunch charge which can exceed well over $100 \mathrm{pC}$ while the beam quality is preserved with the beam characteristics given in Table I. At this point a remark should be made that concerns another major challenge to meet. The FEL interaction involving the computed high extraction efficiency creates a momentum spread in the exhausted beam that exceeds $30 \%$ (full). An ERL FEL has to cope with this large energy spread in transporting the spent beam following the interaction section, the return arc providing the energy acceptance required for a successful beam recovery. The above-mentioned increase in the beam energy, ideally accompanied with an increase in the peak current, would help reduce the (fractional) momentum spread generated in the spent beam (while sustaining the required multi-mJ level 
optical pulse energy output) relaxing hereby the energy acceptance requirements posed on the return arc.

\section{CONCLUSIONS}

In summary, $100 \mathrm{MeV}$ range superconducting ERL driven mid-IR FELs hold great promise in filling a unique niche for generating multi-mJ level, ultrashort ( $\leq 10$ cycles) pulses tunable within the entire mid-IR region (and beyond) with at least many tens of $\mathrm{MHz}$ repetition rates. Because of their ability in providing high peak intensities with excellent temporal and transversal coherence characteristics at unprecedented high repetition rates across the entire NIR/MIR spectral range, they have the potential to become attractive tools in various strong field applications alone or in combination with high finesse enhancement cavities.

In the presented study, a novel high-gain FEL oscillator scheme is investigated. According to the used FEL modeling, for the given system parameters the injection-locked FEL oscillator system operating at zero cavity detuning meets the requirements imposed on a mid-IR drive laser by the up-frequency conversion processes such as HHG to produce attosecond range ultrashort $\mathrm{x}$-ray pulse trains. For being a relatively compact coherent $\mathrm{x}$-ray source, it has the potential of offering a reasonably competitive performance output in terms of average brightness, reach of attosecond time scale, and a broad spectral tuning range as compared to some of its x-ray FEL counterparts consisting of complex, $\mathrm{GeV}$ scale system components.

\section{ACKNOWLEDGMENTS}

The author thanks A. Foehlisch and K. Holldack who have raised the question whether BERLinPro ERL can be employed as a midinfrared FEL driver for HHG that has initiated the study presented in Ref. [21]. He appreciates the useful conversation with A. Baltuska regarding mid-IR OPCPA systems and acknowledges that Kapteyn/Murnane Group was considering to employ midinfrared FEL as a driver laser for $\mathrm{HHG}$ to generate coherent $\mathrm{keV}$ photonenergy x-ray pulses.

Note added in proof.-While this paper was in review, a study has been published [C. Bruni, T. Legrand, C. Szwaj, S. Bielawski, and M. E. Couprie, Phys. Rev. A 84, 063804 (2011)] reporting about the "equivalence between free-electron-laser oscillators and actively mode-locked lasers." Here, in this paper, the claimed mode-locking process of frequency combs relies on a self-starting and self-sustaining phase, amplitude (loss) modulation. The passive mode-locking process that leads to the formation of the described ultrashort, ultrabroad bandwidth, chirped optical pulses in the master oscillator is supported and sustained, as stated in the paper, by the energy transfer from the radiation pulses to the particles that reached the bottom of the ponderomotive potential well as they execute synchrotron oscillations. Assumed fast saturable absorbers as nonlinear loss elements are also included in modeling the master FEL oscillator (standalone) operating at various cavity detuning settings. The mode-locking process exploited in the presented study, however, performs overwhelmingly superior to the latter in terms of pulse shape stability, attained high radiation intensity, and short pulse duration. A detailed account of the study that relates the FEL dynamics described in this paper to mode locking in ultrafast optics will be reported separately.

We also became aware of publications [58,59] that we find instructive for the schemes presented in this paper and in Ref. [23]. It would be in turn interesting to find out whether analogous coupled, injection-locked oscillator configurations can be implemented in other electron beam based radiation sources.

[1] A. Baltuska et al., Nature (London) 421, 611 (2003).

[2] W. Fawley, Nucl. Instrum. Methods Phys. Res., Sect. A 593, 111 (2008).

[3] E. L. Saldin et al., Phys. Rev. ST Accel. Beams 9, 050702 (2006).

[4] A. Zholents, Phys. Rev. ST Accel. Beams 8, 040701 (2005).

[5] D. Xiang et al., Phys. Rev. Lett. 105, 114801 (2010).

[6] I. P. S. Martin and R. Bartolini, Phys. Rev. ST Accel. Beams 14, 030702 (2011).

[7] J. Margos et al., NLS Conceptual Design Report, 2010.

[8] M.-C. Chen et al., Phys. Rev. Lett. 105, 173901 (2010).

[9] T. Popmintchev et al., Nature Photon. 4, 822 (2010).

[10] J. Seres et al., Nature Phys. 6, 455 (2010).

[11] L. Gallman, Nature Phys. 6, 406 (2010).

[12] G. Andriukaitis,T. Balčiūnas, S. Ališauskas, A. Pugžlys, A. Baltuška, T. Popmintchev, M. C. Chen, M. M. Murnane, and H. C. Kapteyn, Opt. Lett. 36, 2755 (2011).

[13] A. Foehlich and K. Holldack (private communication).

[14] A. Baltuska (private communication).

[15] G. Neil et al., Nucl. Instrum. Methods Phys. Res., Sect. A 557, 9 (2006).

[16] H.P. Freund et al., Phys. Rev. Lett. 102, 244802 (2009).

[17] R. J. Jones et al., Phys. Rev. Lett. 94, 193201 (2005).

[18] I. Pupeza, Opt. Lett. 35, 2052 (2010).

[19] J. Paul et al., Opt. Lett. 33, 2482 (2008).

[20] P. Haar, Ph.D. dissertation, Stanford University, 1997.

[21] M. Tecimer, Internal Report HZB, May 1, 2011.

[22] S. Zhang et al., in Proceedings of the 31st International Free Electron Laser Conference (FEL 09), Liverpool, UK (STFC Daresbury Laboratory, Warrington, 2009), p. 211.

[23] G. Svets and J.S. Wurtele, Phys. Rev. E 56, 3606 (1997).

[24] A. E. Siegman, Lasers (University Science Books, Mill Valley, CA, 1986).

[25] S.A. Shakir and W.W. Chow, Phys. Rev. A 32, 983 (1985). 
[26] M. B. Spencer and W.E. Lamb, Jr., Phys. Rev. A 5, 893 (1972).

[27] R. Hajima, N. Nishimori, R. Nagai, and E. J. Minehara, Nucl. Instrum. Methods Phys. Res., Sect. A 475, 270 (2001).

[28] N. Nishimori, R. Hajima, R. Nagai, and E. J. Minehara, Phys. Rev. Lett. 86, 5707 (2001).

[29] R. Hajima and R. Nagai, Phys. Rev. Lett. 91, 5707 (2003).

[30] N. Nishimori, Phys. Rev. E 74, 036502 (2006).

[31] R. Bonifacio et al., Phys. Rev. Lett. 73, 70 (1994).

[32] R. J. Jones et al., Opt. Commun. 175, 409 (2000).

[33] J. Rauschenberger, Ph.D. dissertation, LudwigMaximlians-Universitaet, 2007.

[34] F.X. Kaertner et al., J. Opt. Soc. Am. B 18, 882 (2001).

[35] A. L. Cavalieri, Phys. Rev. Lett. 94, 114801 (2005).

[36] F. Loehl et al., Phys. Rev. Lett. 104, 144801 (2010).

[37] J. Kim and F. X. Kaertner, Laser Photon. Rev. 4, 432 (2010).

[38] M. Tecimer et al., Nucl. Instrum. Methods Phys. Res., Sect. A 528, 139 (2004).

[39] M. Tecimer, Ph.D. dissertation, Tel Aviv University, 2004.

[40] N. M. Kroll et al., IEEE J. Quantum Electron. 17, 1436 (1981).

[41] G. H.C. van Werkhoven et al., Phys. Rev. E 50, 4063 (1994).

[42] B. Levush and T. M. Antonsen, Nucl. Instrum. Methods Phys. Res., Sect. A 285, 136 (1989).
[43] J. Gallardo and L. R. Elias, Nucl. Instrum. Methods Phys. Res., Sect. A 250, 438 (1986).

[44] B.W.J. Mc Neil et al., Opt. Commun. 165, 65 (1999).

[45] C. Penman and B. W. J. Mc Neil, Opt. Commun. 90, 82 (1992).

[46] T. Watanabe et al., Phys. Rev. Lett. 98, 034802 (2007), and references therein.

[47] Bonifacio et al., Phys. Rev. Lett. 99, 029501 (2007), and references therein.

[48] T. Watanabe et al., Phys. Rev. Lett. 99, 029502 (2007).

[49] C. J. Buczek, R. J. Freiberg, and M. L. Scolnick, Proc. IEEE 61, 1411 (1973).

[50] H. Erzgraeber, S. Wieczorek, and B. Krauskopf, Phys. Rev. E 81, 056201 (2010), and references therein.

[51] Y. Kuramoto, Chemical Oscillations, Waves and Turbulence (Springer, Berlin, 1984).

[52] P.C. Matthews, R.E. Mirollo, and S. Strogatz, Physica (Amsterdam) 52D, 293 (1991).

[53] R. Lang and K. Kobayashi, IEEE J. Quantum Electron. 16, 347 (1980).

[54] F. R. Ruiz-Oliveras et al., Nonlin. Sci. Lett. B 1, 69 (2011).

[55] Y. Pinhasi and Yu. Lurie, Phys. Rev. E 65, 026501 (2002).

[56] N. Piovella, Phys. Plasmas 6, 3358 (1999).

[57] M. Kolesik, P. T. Whalen, and J. V. Moloney, IEEE J. Sel. Top. Quantum Electron. 99, 1 (2011).

[58] W. M. Manheimer, B. Levush, and T. M. Antonsen, IEEE Trans. Plasma Sci. 18, 350 (1990).

[59] W. M. Manheimer, Int. J. Electron. 63, 29 (1987). 